\title{
Transient magnetized plasma as an optical element for high power laser pulses
}

\author{
Nobuhiko Nakanii, ${ }^{1,2, *}$ Tomonao Hosokai, ${ }^{1,2,3}$ Kenta Iwasa, ${ }^{3}$ Shinichi Masuda, ${ }^{1,2}$ \\ Alexei Zhidkov, ${ }^{1,2}$ Naveen Pathak, ${ }^{1,2}$ Hiroki Nakahara, ${ }^{3}$ Yoshio Mizuta, ${ }^{3}$ \\ Naoki Takeguchi, ${ }^{3}$ and Ryosuke Kodama ${ }^{1,3,4}$ \\ ${ }^{1}$ Photon Pioneers Center, Osaka University, 2-1 Yamada-oka, Suita, Osaka 565-0871, Japan \\ ${ }^{2}$ CREST, Japan Science and Technology Agency, 2-1 Yamada-oka, Suita, Osaka 565-0871, Japan \\ ${ }^{3}$ Graduate School of Engineering, Osaka University, 2-1 Yamada-oka, Suita, Osaka 565-0871, Japan \\ ${ }^{4}$ Institute of Laser Engineering, Osaka University, 2-8 Yamada-oka, Suita, Osaka 565-0871, Japan
}

(Received 9 October 2014; published 24 February 2015)

\begin{abstract}
Underdense plasma produced in gas jets by low intensity laser prepulses in the presence of a static magnetic field, $B \sim 0.3 \mathrm{~T}$, is shown experimentally to become an optical element allowing steering of tightly focused high power femtosecond laser pulses within several degrees along with essential enhancement of pulse's focusability. Strong laser prepulses form a density ramp perpendicularly to magnetic field direction and, owing to the light refraction, main laser pulses propagate along the magnetic field even if it is tilted from the laser axis. Electrons generated in the laser pulse wake are well collimated and follow in the direction of the magnetic field; their characteristics are measured to be not sensitive to the tilt of magnetic field up to angles $\pm 5^{\circ}$.
\end{abstract}

DOI: 10.1103/PhysRevSTAB.18.021303

PACS numbers: 52.38.Kd, 41.75.Jv

\section{INTRODUCTION}

Consistent control and steering of high intensity laser pulses is an important requirement for experimental research in fields such as laser wakefield acceleration [1-3], plasma photonics [4], high-energy density physics [5-7], and so on to provide stable, repeatable results of measurements. The conventional optics may be not able to support required guiding and steering of high-energy particles or high power laser pulses. Plasma is the most prominent object as a nondestructive optical element for such a purpose, both for charged particles [8-10] and for high power laser beams [11-18].

Laser-wakefield accelerators with the internal particle injection (self-injection) could become a basis for fulloptical, high-temporal and high-spatial resolution imaging systems [19-21] as well as the first stage in the ultrahighenergy multistage acceleration technique [11,22]. In case of electrons, applications of laser-driven electron bunches require their high charges, low emittances, low-energy spreads, and also high reproducibility. These parameters are very sensitive to the plasma and laser conditions. The optical steering here is one of the most important technique allowing high stability and controllability of the laser wakefield acceleration.

*nobu.nakanii@ppc.osaka-u.ac.jp

Published by the American Physical Society under the terms of the Creative Commons Attribution 3.0 License. Further distribution of this work must maintain attribution to the author $(s)$ and the published article's title, journal citation, and DOI.
Plasma optics for high power, femtosecond laser pulses can be based on the optical property of preplasma produced by prepulses via optical field ionization or optical breakdown [12,15-18]. It is well known that focusing, defocusing, and/or scattering of light in plasma depends on the electron density gradient $\nabla n(\mathbf{r}, \omega)=$ $-\nabla\left[N_{e} / N_{\mathrm{cr}}(\omega)\right] / n(\mathbf{r}, \omega)$, where $n(\mathbf{r}, \omega)$ is the refraction index, $N_{\mathrm{cr}}=m \omega^{2} / 4 \pi e^{2}$ the critical density and $\omega$ the light frequency. In the frame of geometrical optics a path of laser light can be found as a minimum of the functional $\Phi\left(\mathbf{r}_{\perp}, d r_{\perp} / d z\right)=\int n(\mathbf{r}, \omega) \times$ $\sqrt{1+\left(d r_{\perp} / d z\right)^{2}} d z$, which results in the equation for the trajectory $d\left[n(\mathbf{r}, \omega) \mathbf{r}_{\perp} / \sqrt{1+\left(d r_{\perp} / d z\right)^{2}}\right] / d z=$ $\sqrt{1+\left(d r_{\perp} / d z\right)^{2}} \nabla_{\perp} n(\mathbf{r}, \omega)$. For a parabolic channel $N_{e}=N_{e 0}\left[1+\delta r_{\perp}^{2} / D^{2}\right]$ and a plane wave $a_{0} e^{i k_{z} z+i k_{x} x}$ the solution of the equation gives the total pulse scattering up to $10^{\circ}$ angle for $N_{e 0}=10^{19} \mathrm{~cm}^{-3}, D=20 \mu \mathrm{m}, \delta=3$. Nonlinear effects such as distortion of electron density distribution by pulse's ponderomotive force and distortion of laser pulse shape do not change such scattering essentially which is confirmed by particle-in-cell (PIC) simulations $[23,24]$. Figure 1(a) illustrates dynamics of a laser pulse field in a semiparabolic plasma channel near a scattering point [25] (see below). Then, the laser pulse is symmetrically reflected and the angle of its propagation is changed from $-\theta$ to $+\theta$. It is apparent that a preplasma may affect the propagation and focusability of main pulse. However, a single scattering of high intensity laser pulses with keeping their profile and energy in an optical element has not been demonstrated experimentally. 
(a)

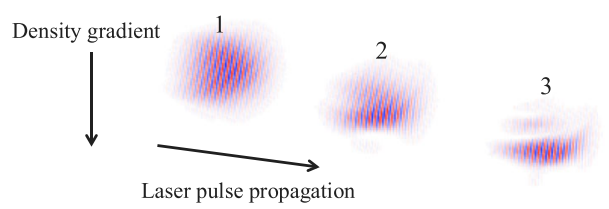

(b)

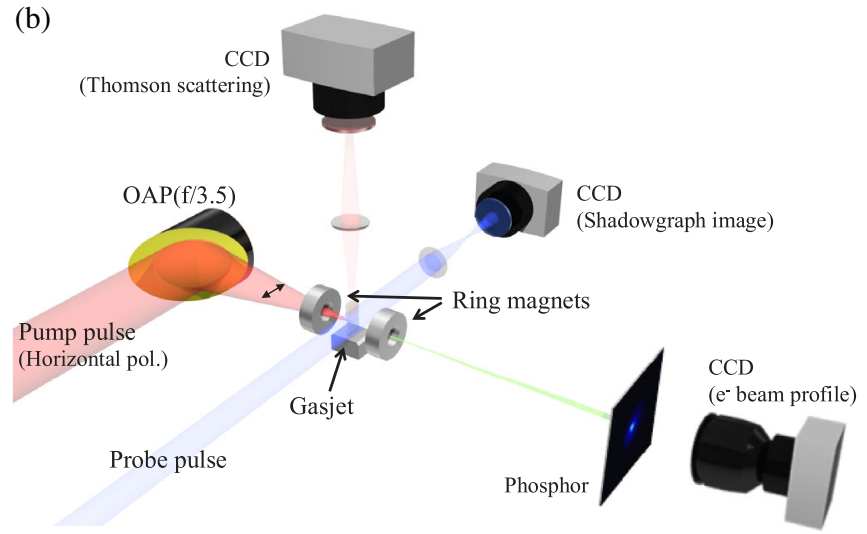

FIG. 1. (a) Dynamics of a laser pulse field in a semiparabolic plasma channel near a scattering point. (b) Experimental setup.

Practically, there are several ways to produce a plasma channel able to guide high power laser pulses [11-18]. Transient plasma generated by a low intensity laser pulse, a splash channel $[11,12]$, is the most appropriate candidate for a plasma optical element. Moreover as demonstrated experimentally in Ref. [26] an external magnetic field, $B \sim 0.1-0.3 \mathrm{~T}$, may result in the formation of preplasmas (splash channels [12]) changing their density gradients and, therefore, in the propagation of powerful laser pulse. In the regime of splash channel formation [12] if the magnetic field is tilted from the laser axis, the density ramp can be formed only by a component of the ponderomotive force perpendicular to the magnetic field direction, and a following main laser pulse starts its propagation as a tilted laser pulse in a plasma channel. We observed a deformation of a splash plasma channel with the gas density $N=$ $3 \times 10^{19} \mathrm{~cm}^{-3}$ in an external magnetic field $B=0.3 \mathrm{~T}$ when plasma is irradiated by a picosecond laser pulse with the intensity $I=10^{14} \mathrm{~W} / \mathrm{cm}^{2}$ focused in $10 \mu \mathrm{m}$ spot via 2D PIC calculation including elastic collisions and optical field ionization [11]. To reveal the tangibility of plasma optics with the use of an external magnetic field, we also performed the 3D PIC simulation of the laser focusing in a transforming plasma cone with the shape $N_{e}=$ $N_{e 0} \times\left(1+a \times r^{2}-b \times y\right) ; a \gg b$. A result of the simulation for $\sim 6^{\circ}$ distortion is shown in Fig. 1(a). The result confirms the change of the direction of the laser pulse propagation during its focusing in the preplasma cone. However, the quality of laser light after its tilting can be found only experimentally.

In this paper, for the first time we experimentally demonstrate the plasma optical element allowing steering and precise control of direction of high-quality ultrashort electron beam via tilting a relatively weak static magnetic field and changing the intensity of laser prepulses.

\section{EXPERIMENTAL SETUP}

The experimental setup is shown in Fig. 1(b). A 40-TW Ti:sapphire laser system (Amplitude Technologies) at Photon Pioneers Center, Osaka University, based on a chirped pulse amplification (CPA) technique can deliver up to $1.0-\mathrm{J}$ pulses at a central wavelength of $800 \mathrm{~nm}$ with a repetition rate of $10 \mathrm{~Hz}$. In this experiment, the pulse energy on target was varied from 180 to $460 \mathrm{~mJ}$ and the pulse duration was $\sim 30 \mathrm{fs}$. The laser irradiation is conducted in single-shot mode with the repetition rate around $0.1 \mathrm{~Hz}$, which is limited by the vacuum pumping speed.

A shock-wave-free slit nozzle was designed for Mach number 5 at the exit for helium. The shape of the nozzle exit is rectangular of $1.2 \mathrm{~mm}$ length and $4.0 \mathrm{~mm}$ width. A horizontally polarized laser beam with a diameter of $\sim 5 \mathrm{~cm}$ is focused on the position of $\sim 100 \mu \mathrm{m}$ from the front edge of the slit nozzle at a height of $\sim 1 \mathrm{~mm}$ from the nozzle exit by a gold-coated off-axis parabolic mirror with $f / 3.5$ $(f=177.8 \mathrm{~mm})$. The gas number densities are estimated to be $1.1-3.4 \times 10^{19} \mathrm{~cm}^{-3}$. The focal spot size on target is $7-8 \mu \mathrm{m}$ of $1 / e^{2}$ diameter. The laser energy within $1 / e^{2}$ diameter is $24 \%$ of the total energy. The maximum laser intensity on the target is estimated to be $1.7 \times 10^{19} \mathrm{~W} / \mathrm{cm}^{2}$; the corresponding normalized vector potential $a_{0}$ is around 2.8. We change the prepulse energy via changing the input energies of seed pulse and pump pulse in regenerative amplification in the CPA system. Prepulse shapes in 100-ps regime were measured by third-order cross correlator (SEQOIA, Amplitude Technology). However, the measurements can be done only for the contrast ratio between the main pulse and the nanosecond prepulse caused by the amplified spontaneous emission (ASE), which was variable from $10^{7}$ to $10^{10}$.

As shown in Fig. 1(b), a magnetic device applying an external static magnetic field in the laser propagation direction consists of two ring-shaped neodymium permanent magnets and the gas jet nozzle is placed between these magnets. One is set in front of and the other is set on the rear side of the nozzle on the laser axis. Laser pulses pass through the hole of the front ring magnet and interact with the gas jet target. The inner diameter, the outer diameter and the thickness of the ring magnet are 8, 18, and $12 \mathrm{~mm}$, respectively. The distance between these two ring magnets is $14 \mathrm{~mm}$. The magnetic field has the strength of $B \sim$ $0.25 \mathrm{~T}$ and is parallel to the laser axis. The axis of the magnetic field can be tilted horizontally and vertically by controlling motorized stages under vacuum condition.

The spatial distribution of ejected electron beams is measured by a phosphor screen (Mitsubishi Chemical Co. LTD, DRZ-High) with a diameter of $13 \mathrm{~cm}$ placed at $25.5 \mathrm{~cm}$ away from the gas jet target or that with a diameter of $9 \mathrm{~cm}$ at $43 \mathrm{~cm}$ from the target. The DRZ-High 
(a)

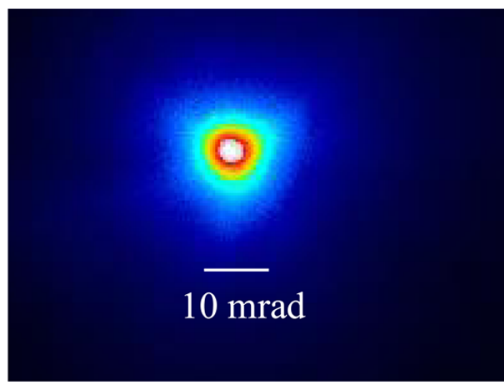

(c)

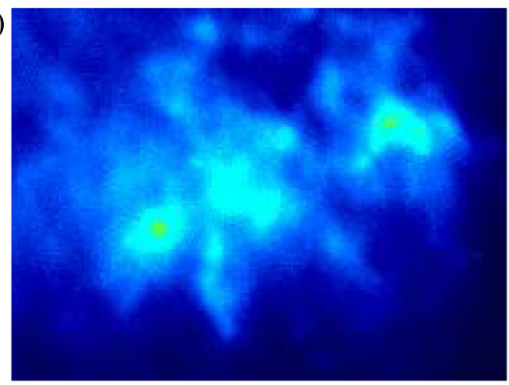

(b)

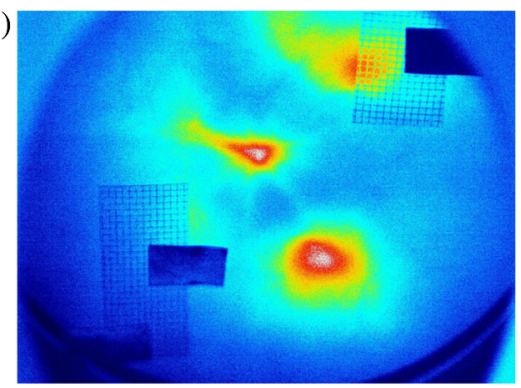

(d)

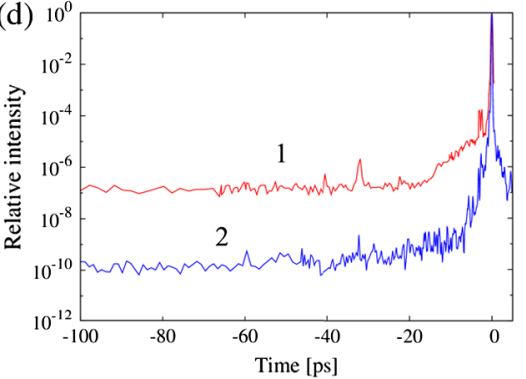

FIG. 2. Electron beam profiles (a) in the case of high-intensity prepulse with the external magnetic field, (b) low-intensity prepulse in the magnetic field, and (c) high-intensity prepulse without the magnetic field for the gas density $\sim 3.4 \times 10^{19} \mathrm{~cm}^{-3}$. (d) Prepulse shapes in hundred ps regime measured by third-order cross correlator.

screen has a $\mathrm{Gd}_{2} \mathrm{O}_{2} \mathrm{~S}$ : $\mathrm{Tb}$ phosphor layer with the emission peak at $545 \mathrm{~nm}$. The screen is sensitive to high-energy particles and radiations, so that the front side of the screen is laminated with a $12 \mu \mathrm{m}$-thick aluminum and $15 \mu \mathrm{m}$-thick titanium foils to avoid exposure to the laser pulses, scattering lights and low-energy electrons. The scintillating images on the screen made by the deposited electrons are recorded by the CCD camera (Bitran Co., BU-51LN) with a commercial photographic lens from the back side of the screen. To reduce the background noise due to scattering light of laser pulses, a green-pass filter (BG-39) was set at the front of the camera.

\section{RESULTS AND DISCUSSIONS}

The effect of laser prepulse in the presence of an external magnetic field is illustrated by Fig. 2 when the direction of magnetic field coincides with the direction of laser pulse propagation. The difference between low and high contrast ratio pulse is clearly seen. The typically stable, well-collimated spatial distribution as in Fig. 2(a) is produced with the highest-intensity prepulse (ASE level $\sim 10^{12} \mathrm{~W} / \mathrm{cm}^{2}$ ), given curve 1 in Fig. 2(d). On the other hand, the spatial profile with a lower-intensity prepulse (ASE level $\sim 10^{9} \mathrm{~W} / \mathrm{cm}^{2}$ ), corresponding to curve 2 in Fig. 2(d), presented in Fig. 2(b) is spotted and typically unstable. There is also a rough and unstable distribution without magnetic field as shown in Fig. 2(c). We attribute this effect to the plasma optical element which optical quality depends on the prepulse intensity: only a high intensity laser prepulse forms a density gradient able to improve a poor laser energy distribution in the focus spot.
The direction of laser pulse propagation can be seen via traces of Thomson scattering. Time-integrated images of Thomson scattering of laser pulse from the plasma with different tilting angles and different intensities of the laser prepulses are shown in Fig. 3. The laser pulse propagates from left to right. The dashed line shows the laser injection axis and the solid arrow shows the tilting magnetic field axis. The bright spots display the points where the Thomson scattering of main pulse is the strong. As seen in Fig. 3(a), in the case of lower intensity prepulses, which have the contrast ratio higher than $10^{8}$ (the corresponding ASE level less than $\sim 10^{11} \mathrm{~W} / \mathrm{cm}^{2}$ ), a tilt of magnetic field (a)

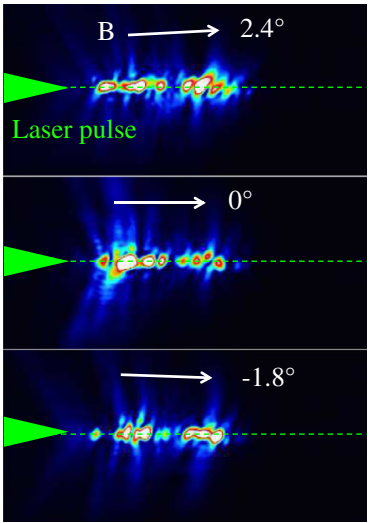

(b)

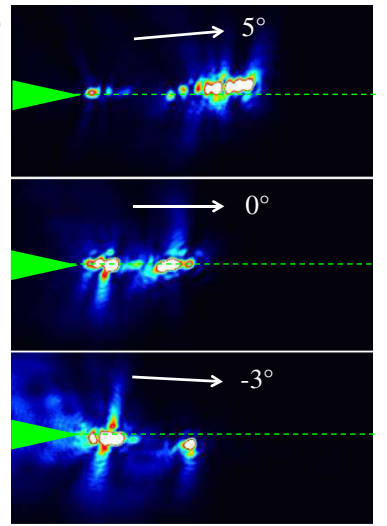

FIG. 3. Time-integrated images of Thomson scattering of laser pulse from the plasma with different tilting angles (a) in case of low-intensity prepulse (ASE level $\sim 10^{11} \mathrm{~W} / \mathrm{cm}^{2}$ ) for the gas density $\sim 1.1 \times 10^{19} \mathrm{~cm}^{-3}$ and (b) high-intensity prepulse (ASE level $\sim 10^{12} \mathrm{~W} / \mathrm{cm}^{2}$ ) for the gas density $\sim 2.3 \times 10^{19} \mathrm{~cm}^{-3}$. 
(a)

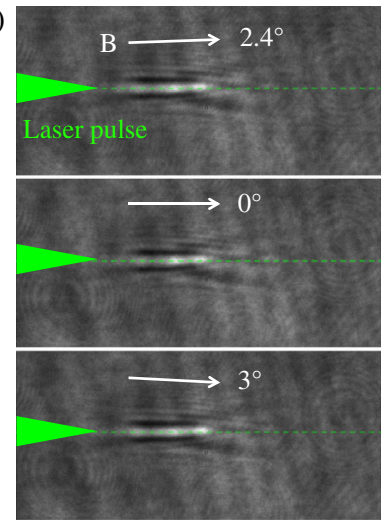

(b)

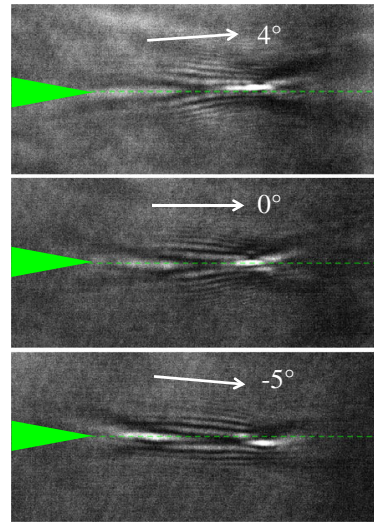

FIG. 4. Shadowgraph images with different tilting angles (a) in the case of low-intensity prepulse (ASE level $\sim 10^{11} \mathrm{~W} / \mathrm{cm}^{2}$ ) for the gas density $\sim 1.1 \times 10^{19} \mathrm{~cm}^{-3}$ and (b) high-intensity prepulse (ASE level $\sim 10^{12} \mathrm{~W} / \mathrm{cm}^{2}$ ) for the gas density $\sim 2.3 \times 10^{19} \mathrm{~cm}^{-3}$.

does not result in the steering of laser pulse propagation. The laser pulses propagate along with the initial laser axis. The structure of hot spots appeared from Thomson scattering is quite stochastic. This means there could be no preplasma formed before the main pulse. In contrast with an increase of intensity of the prepulse as curve 1 in Fig. 2(d), directions of laser beam propagation exactly follow the directions of the magnetic even if it tilted $5^{\circ}$ from the initial laser axis as shown in Fig. 3(b). In the present experiment, $5^{\circ}$ was a maximal tilt degree allowed by our setup. With the strongest prepulse the main pulses exactly follow the direction of magnetic field within $-5^{\circ}$ to $+5^{\circ}$. A similar tendency is observed in the shadowgraph images presented in Fig. 4. Similar to the Thomson scattering, the plasma channels are directed along the magnetic field even if it tilted from the laser axis in the case of strong prepulses and follow the laser axis when the prepulse intensity is weak to form the optical element. The shadowgraph image in Fig. 4 is not the exact image of the plasma channel because the halo, which is the outside part of the focal spot caused by the poor wave front, is less affected by the plasma optics and, therefore, creates a larger and slightly less tilted image.

There should be threshold intensity when a main pulse will break through the splash plasma channel due to the ponderomotive force. In the present experiment the laser intensity was limited by $I_{\max }=2 \times 10^{19} \mathrm{~W} / \mathrm{cm}^{2}$ that is far lower than the threshold. It should be also a density threshold. At too low density the refraction effect stops working. In the present setup the signals from plasma with its density $N \sim 5 \times$ $10^{18} \mathrm{~cm}^{-3}$ were too low for conclusive detection. Therefore we were not able to find the density threshold.

The quality of the scattered laser light can be characterized by the process of laser wakefield acceleration. This process consists of two parts: (i) electron self-injection and (ii) electron acceleration by laser wakefield. The first part determines the total charge of accelerated electrons, the second their energy distribution. It is clear that if the quality of the scattered light is poor further electron acceleration becomes impossible due to corresponding poor quality of the laser pulse wake. In contrast, if the quality of the scattered light is good the electron acceleration in the tilted magnetic field should run similarly to the case $\theta=0^{\circ}$. Electron beam profiles on the phosphor screen obtained with the low contrast ratios of $10^{7}$ (the corresponding intensity of the ASE $\sim 10^{12} \mathrm{~W} / \mathrm{cm}^{2}$ ) and with tilted static magnetic field are shown in Fig. 5(a). One can see that the well-collimated accelerated electron also exactly follows the direction of the magnetic field in Fig. 5(b). The pointing stability of electron bunches in this case is very high: the beam divergence is $\sim 11 \mathrm{mrad}$ in full angle; the pointing stability is less than $0.5 \mathrm{mrad} \mathrm{rms}$ in each tilt angle of the magnetic field.

The total charge and the energy distribution of accelerated electrons for different tilt angles are given in Fig. 6. Within typical experimental fluctuations of the total charge its value does not depend on the angle of the tilted magnetic field, as in Fig. 6(a). The energy distribution of accelerated (a)

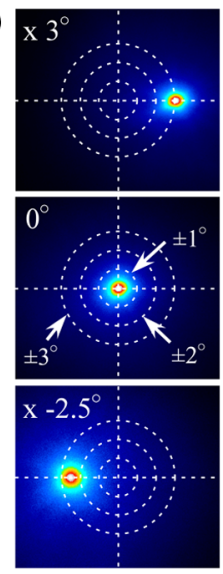

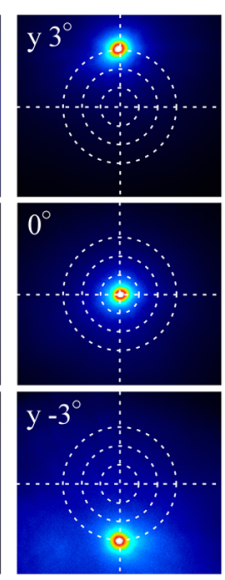

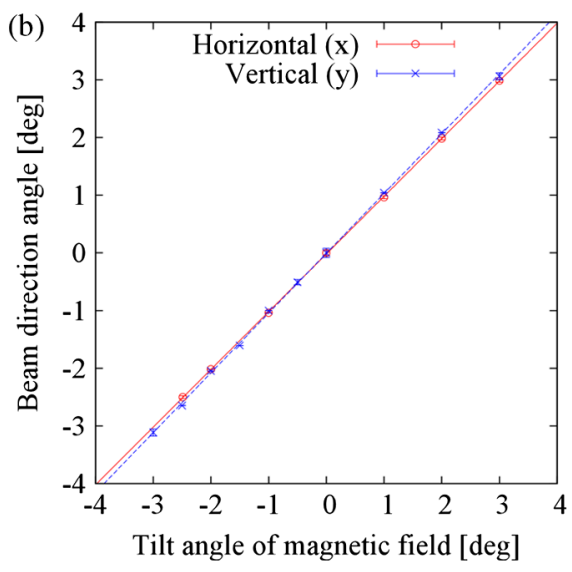

Tilt angle of magnetic field [deg]

FIG. 5. (a) Electron beam profiles on the phosphor screen and (b) the beam pointing obtained by irradiating the laser pulse with the high-intensity prepulse (ASE level $\sim 10^{12} \mathrm{~W} / \mathrm{cm}^{2}$ ) into the gas jet with the density $\sim 4.5 \times 10^{19} \mathrm{~cm}^{-3}$ under tilted static magnetic field. 

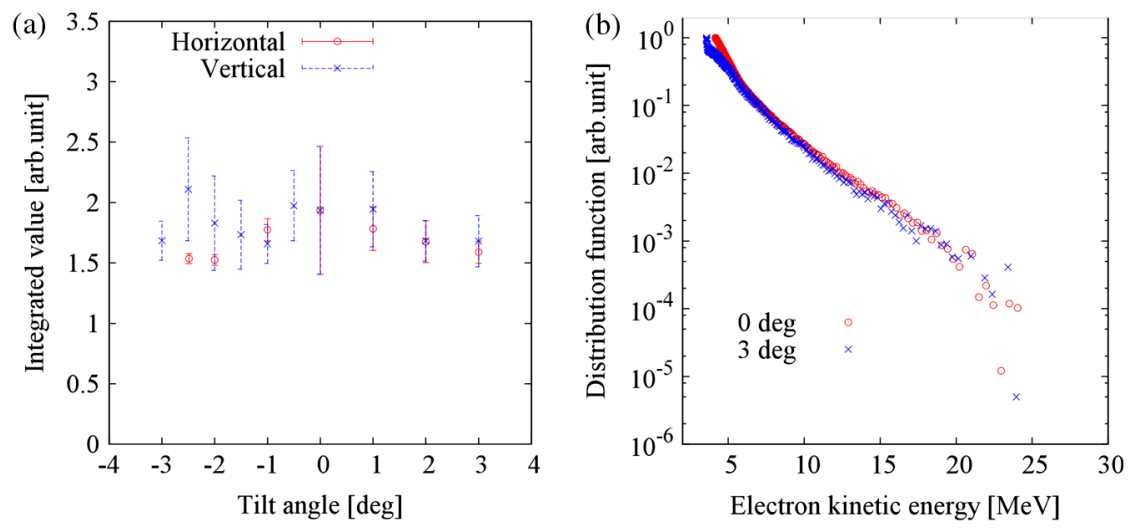

FIG. 6. (a) Total charge and (b) energy distribution of accelerated electrons for different tilt angles of the magnetic field.

electrons is not sensitive to the tilt angle as shown in Fig. 6(b). These results confirm that the processes of electron self-injection and laser wakefield acceleration run similarly when the main laser changes its direction of propagation in the plasma optical element.

\section{CONCLUSION}

In conclusion, we have demonstrated experimentally that a high power laser pulse can be controllably directed by tilting a static magnetic field. We have shown that if intensity of laser prepulse is enough it produces a preplasma with the density gradient perpendicular to the direction of an external magnetic field. If the direction of magnetic field is tilted from the laser axis the main laser pulse starts propagating as if it is incident on the plasma at a certain angle. After scattering at the density gradient the pulse propagates in the direction of the magnetic field. The steered laser pulse generates the wake similar to that produced by a laser pulse propagating at $0^{\circ}$ angle. The wave-breaking process, electron self-injection, and electron acceleration run similarly and give the identical accelerated electron bunches demonstrating ability of precise direction control and steering of electrons in laser wakefield acceleration.

\section{ACKNOWLEDGMENTS}

The authors thank Dr. M. Kando, Dr. H. Kotaki, and Dr. M. Mori at KPSI, JAEA for useful discussions and technical support. This work was supported by Core Research of Evolutional Science and Technology (CREST) of Japan Science and Technology Agency (JST). It was also supported by Genesis Research Institute, Inc. (Konpon-ken, TOYOTA).

[1] T. Tajima and J. M. Dawson, Phys. Rev. Lett. 43, 267 (1979).

[2] E. Miura, K. Koyama, S. Kato, N. Saito, M. Adachi, Y. Kawada, T. Nakamura, and M. Tanimoto, Appl. Phys. Lett. 86, 251501 (2005); S. P. D. Mangles, C. D. Murphy,
Z. Najmudin, A. G. R. Thomas, J. L. Collier, A. Dangor, P. S. Foster, J. L. Collier, E. J. Divall, J. G. Gallacher, C. J. Hooker, D. A. Jaroszynski, A. J. Langley, W. B. Mori, P. A. Norreys, F. S. Tsung, R. Viskup, B. R. Walton, and K. Krushelnick, Nature (London) 431, 535 (2004); C. G. R. Geddes, C. Toth, J. van Tilborg, E. Esarey, C. B. Schroeder, D. Bruhwiler, C. Nieter, J. Cary, and W. P. Leemans, Nature (London) 431, 538 (2004); J. Faure, Y. Glinec, A. Pukhov, S. Kiselev, S. Gordienko, E. Lefebvre, J.-P. Rousseau, F. Burgy, and V. Malka, Nature (London) 431, 541 (2004).

[3] W. P. Leemans, B. Nagler, A. J. Gonsalves, C. Toth, K. Nakamura, C. G. R. Geddes, E. Esarey, C. B. Schroeder, and S. M. Hooker, Nat. Phys. 2, 696 (2006); S. Karsch, J. Osterhoff, A. Popp, T. P. Rowlands-Rees, Z. Major, M. Fuchs, B. Marx, R. Horlein, K. Schmid, L. Veisz, S. Becker, U. Schramm, B. Hidding, G. Pretzler, D. Habs, F. Gruner, F. Krausz, and S. M. Hooker, New J. Phys. 9, 415 (2007); X. Wang et al., Nat. Commun. 4, 1988 (2013).

[4] R. Kodama et al., Nature (London) 432, 1005 (2004).

[5] T. Ditmire, S. Bless, G. Dyer, A. Edens, W. Grigsby, G. Hays, K. Madison, A. Maltsev, J. Colvin, M. J. Edwards, R. W. Lee, P. Patel, D. Price, B. A. Remington, R. Shepperd, A. Wotton, J. Zweiback, E. Liang, and K. Keilty, Radiat. Phys. Chem. 70, 535 (2004).

[6] G. Mourou, T. Tajima, and S. V. Bulanov, Rev. Mod. Phys. 78, 309 (2006).

[7] S. A. Pikuz, A. Y. Faenov, J. Colgan, R. J. Dance, J. Abdallah, E. Wagenaars, N. Booth, O. Culfa, R. G. Evans, R. J. Gray, T. Kaempfer, K. L. Lancaster, P. McKenna, A. Rossall, I. Y. Skobelev, K. S. Schulze, I. Uschmann, A. G. Zhidkov, and N. C. Woolsey, High Energy Density Phys. 9, 560 (2013).

[8] P. Muggli, S. Lee, T. Katsouleas, R. Assmann, F.-J. Decker, M. J. Hogan, R. Iverson, P. Raimondi, R. H. Siemann, D. Walz, B. Blue, C. E. Clayton, E. Dodd, R. Fonseca, R. Hemker, C. Joshi, K. A. Marsh, W. B. Mori, and S. Wang, Nature (London) 411, 43 (2001).

[9] R. Weingartner, M. Fuchs, A. Popp, S. Raith, S. Becker, S. Chou, M. Heigoldt, K. Khrennikov, J. Wenz, T. Seggebrock, B. Zeitler, Z. Major, J. Osterhoff, F. Krausz, S. Karsch, and F. Gruner, Phys. Rev. ST Accel. Beams 14, 052801 (2011). 
[10] T. Toncian, M. Borghesi, J. Fuchs, E. dHumieres, P. Antici, P. Audebert, E. Brambrink, C. A. Cecchetti, A. Pipahl, L. Romagnani, and O. Willi, Science 312, 410 (2006).

[11] T. Hosokai, A. Zhidkov, A. Yamazaki, Y. Mizuta, M. Uesaka, and R. Kodama, Appl. Phys. Lett. 96, 121501 (2010).

[12] Y. Mizuta, T. Hosokai, S. Masuda, A. Zhidkov, K. Makito, N. Nakanii, S. Kajino, A. Nishida, M. Kando, M. Mori, H. Kotaki, Y. Hayashi, S. V. Bulanov, and R. Kodama, Phys. Rev. ST Accel. Beams 15, 121301 (2012).

[13] Y. Kitagawa, Y. Sentoku, S. Akamatsu, W. Sakamoto, R. Kodama, K. A. Tanaka, K. Azumi, T. Norimatsu, T. Matsuoka, H. Fujita, and H. Yoshida, Phys. Rev. Lett. 92, 205002 (2004); F. Dorchies, J. R. Marques, B. Cros, G. Matthieussent, C. Courtois, T. Velikoroussov, P. Audebert, J. P. Geindre, S. Rebibo, G. Hamoniaux, and F. Amiranoff, Phys. Rev. Lett. 82, 4655 (1999).

[14] Y. Ehrlich, C. Cohen, A. Zigler, J. Krall, P. Sprangle, and E. Esarey, Phys. Rev. Lett. 77, 4186 (1996); T. Hosokai, M. Kando, H. Dewa, H. Kotaki, S. Kondo, N. Hasegawa, K. Nakajima, and K. Horioka, Opt. Lett. 25, 10 (2000); A. Butler, D. J. Spence, and S. M. Hooker, Phys. Rev. Lett. 89, 185003 (2002); T. Kameshima, H. Kotaki, M. Kando, I. Daito, K. Kawase, Y. Fukuda, L. M. Chan, T. Homma, S. Kondo, T. Z. Esirkepov, N. A. Bobrova, P. V. Sasorov, and S. V. Bulanov, Phys. Plasmas 16, 093101 (2009).

[15] C. G. Durfee and H. M. Milchberg, Phys. Rev. Lett. 71, 2409 (1993).

[16] T. R. Clark and H. M. Milchberg, Phys. Rev. E 61, 1954 (2000); T. R. Clark and H. M. Milchberg, Phys. Rev. Lett. 78, 2373 (1997); V. Malka, E. DeWispelaere, F. Amiranoff, S. Baton, R. Bonadio, C. Coulaud, R. Haroutunian, A. Modena, D. Puissant, C. Stenz, S. Huller, and M. Casanov, Phys. Rev. Lett. 79, 2979 (1997); J. Faure, V. Malka, J.-R. Marques, F. Amiranoff, C. Courtois, Z. Najmudin, K. Krushelnick, M. Salvati, A. Dangor, A. Solodov, P. Mora, J.-C. Adam, and A. Heron, Phys. Plasmas 7, 3009 (2000).

[17] K. Krushelnick, A. Ting, C. I. Moore, H. R. Burris, E. Esarey, P. Sprangle, and M. Baine, Phys. Rev. Lett. 78,
4047 (1997); S.-Y. Chen, G. S. Sarkisov, A. Maksimchuk, R. Wagner, and D. Umstadter, Phys. Rev. Lett. 80, 2610 (1998).

[18] A. Giulietti, P. Tomassini, M. Galimberti, D. Giulietti, L. A. Gizzi, P. Koester, L. Labate, T. Ceccotti, P. DOliveria, T. Auguste, P. Monot, and P. Martin, Phys. Plasmas 13, 093103 (2006); J. Faure, C. Rechatin, O. Lundh, L. Ammoura, and V. Malka, Phys. Plasmas 17, 083107 (2010).

[19] S. Tokita, M. Hashida, S. Inoue, T. Nishoji, K. Otani, and S. Sakabe, Phys. Rev. Lett. 105, 215004 (2010).

[20] Z. H. He, A. G. R. Thomas, B. Beaurepaire, J. A. Nees, B. Hou, V. Malka, K. Krushelnick, and J. Faure, Appl. Phys. Lett. 102, 064104 (2013).

[21] W. Schumaker, N. Nakanii, C. McGuffey, C. Zulick, V. Chyvkov, F. Dollar, H. Habara, G. Kalintchenko, A. Maksimchuk, K. A. Tanaka, A. G. R. Thomas, V. Yanovsky, and K. Krushelnick, Phys. Rev. Lett. 110, 015003 (2013).

[22] D. Kaganovich, A. Ting, D. F. Gordon, R. F. Hubbard, T. G. Jones, A. Zigler, and P. Sprangle, Phys. Plasmas 12, 100702 (2005).

[23] T. Ohkubo, S. V. Bulanov, A. G. Zhidkov, T. Esirkepov, J. Koga, M. Uesaka, and T. Tajima, Phys. Plasmas 13, 103101 (2006).

[24] A. Popp, J. Vieira, J. Osterhoff, Z. Major, R. Horlein, M. Fuchs, R. Weingartner, T. P. Rowlands-Rees, M. Marti, R. A. Fonseca, S. F. Martins, L. O. Silva, S. M. Hooker, F. Krausz, F. Gruner, and S. Karsch, Phys. Rev. Lett. 105, 215001 (2010).

[25] Results of two-dimensional particle-in-cell simulation with the arbitrary moving window technique, the pulse intensity $I=3 \times 10^{19} \mathrm{~W} / \mathrm{cm}^{2}$, pulse duration $\tau=20 \mathrm{fs,}$ pulse waist $w_{0}=5 \mu \mathrm{m}$, a parabolic electron density profile, $N_{e}=N_{e \min }+\left(N_{e \max }-N_{e \min }\right)\left[\eta\left(y-y_{0}\right)\right]^{2} / D^{2}$ with $N_{e \min }=$ $1 \times 10^{19} \mathrm{~cm}^{-3}, N_{e \max }=3 \times 10^{19} \mathrm{~cm}^{-3}$, and $D=40 \mu \mathrm{m}$.

[26] T. Hosokai, K. Kinoshita, A. Zhidkov, A. Maekawa, A. Yamazaki, and M. Uesaka, Phys. Rev. Lett. 97, 075004 (2006). 